\title{
Cardiomioplastia: novo gerador da Biotronic
}

\author{
Domingo M. BRAILE*, Marcelo J. F. SOARES*, Dorotéia R. S. SOUZA*, Max SCHALDACH ${ }^{\star *}$
}

RBCCV 44205-230

BRAILE, D. M.; SOARES, M. J. F.; SOUZA, D. R. S.; SCHALDACH, M. - Cardiomioplastia: novo gerador da Biotronic. Rev. Bras. Cir. Cardiovasc., 9(1): 40-53, 1994.

RESUMO: Na cardiomioplastia, a contração do músculo esquelético, submetido à estimulaçāo elétrica sobre a parede ventricular dilatada, aumenta a função ventricular, que é dependente das condiçōes prévias do coração e da doença de base. Um dos problemas principais que interfere no sucesso da substituição do músculo cardiaco é a estimulação sincrônica do miocárdio e o músculo esquelético. A estimulaçăo desse músculo a longo prazo tem sido possível graças a eletrodos especiais associados à estimulaçăo progressiva seqūencial, adaptando-o à função cardiaca, através da transformação gradual de fibras glicolíticas expostas à fadiga em oxidativas lentas altamente resistentes. O gerador de pulsos "Myos" (Biotronik) tem sido utilizado em nosso Serviço para estimulação elétrica do músculo grande dorsal em sincronização com o miocárdio. Esse tipo de cardiomioestimulador com circuito eletrônico e bateria de lítio armazena um programa de estimulação responsável por diferentes modos operacionais, adaptados por um programa de computação. Para a programaçāo do cardiomioestimulador, o momento da sincronizaçăo do trem de pulso com a abertura da valva aórtica é de extrema importância. O modo $\mathrm{M}$ de alta velocidade é utilizado para avaliar este sincronismo. A avaliação clínica da cardiomioplastia tem como base os resultados obtidos de 32 pacientes, com idade de 22 a 72 anos (média $=46,2$ anos). A maioria $(72 \%)$ dos pacientes apresentou miocardiopatia dilatada por causa indeterminada, $24 \%$ de origem chagásica, $3 \%$ virótica e $3 \%$ por periparto. Os indices de mortalidade hospitalar e tardia foram ambos de $12,5 \%$ e de $3,1 \%$, respectivamente, excluindo-se os chagásicos. A sobrevida atuarial foi de $81,3+-0,22 \%$ após 6 anos e de $94,4+-0,1 \%$ após 5 anos, retirando-se os chagásicos. Os índices médios de diâmetros sistólico $(55,1 \mathrm{~mm})$, diastólico $(70,7 \mathrm{~mm})$, encurtamento segmentar $(22,8 \%)$ e fração de ejeção $(51,0 \%)$, referentes a seguimento médio de 14,2 meses, refletem que a cardiomioplastia pode ser efetiva na assistência do ventrículo esquerdo. A escolha do paciente parece ser a chave para o bom resultado operatório a curto e longo prazos.

DESCRITORES: cardiomioplastia; cardiomioestimulador; músculo grande dorsal.

\section{INTRODUÇÃO}

A cardiomioplastia foi pioneiramente introduzida por KANTROWITZ \& MCKINNON ${ }^{25}$ e HUME ${ }^{23}$, nas décadas de 50 e 60 , e posteriormente desenvolvida em humanos por CARPENTIER \& CHACHQUES ${ }^{8}$. Esse procedimento ultrapassa a simples transposição da massa muscular da parede torácica para o miocárdio. Os enxertos sem estimulação são insuficientes e, se desnervados, logo atrofiam 21 e tornam-se rígidos ${ }^{37}$; pelo contrário, esse tipo de assistência ventricular biomecânica consiste de um sistema de estimulação elétrica seqüencial progressiva, capaz de condicionar o músculo esquelético tornando-o resistente à fadiga, beneficiando pacientes com cardiomiopatia isquêmica e dilatada $1,7,8$, $11,20,30,32$.

O músculo esquelético pode desenvolver tensões de 1 a $5 \mathrm{~kg} / \mathrm{cm}^{2}$, o que o torna mais do que suficiente para auxiliar o coração no bombeamento do sangue, uma vez que a tensão nominal desenvolvida por este não passa de $0,5 \mathrm{~kg} / \mathrm{cm}^{2}$.

Alguns princípios básicos, contudo, têm que ser considerados como a lei de Laplace, em que a tensão é proporcional ao quadrado do raio 4,42 . Desta forma, quanto maior for o diâmetro do ventrículo a ser

Trabalho realizado na Faculdade de Medicina de São José do Rio Preto e na Unicamp, Brasil.

Apresentado ao $21^{\circ}$ Congresso Nacional de Cirurgia Cardiaca. Porto Alegre, 24 a 26 de março, 1994.

- De Braile Cardiocirurgia, da Faculdade de Medicina de São José do Rio Preto e da Unicamp.

* Da Universidade de Erlagen. Alemanha.

Endereço para correspondência: Av. Juscelino Kubitschek, 3101. 1509-450 São José do Rio Preto, SP, Brasil. 
BRAILE, D. M.; SOARES, M. J. F.; SOUZA, D. R. S.; SCHALDACH, M. - Cardiomioplastia: novo gerador da Biotronic. Rev. Bras. Cir. Cardiovasc., $9(1): 40-53,1994$.

en-volvido pelo músculo, maior será a tensão a ser desenvolvida pelo mesmo, podendo ultrapassar o seu limite.

Outro princípio básico a ser considerado é o de que a contração do músculo esquelético não responde à lei do tudo ou nada como no coração, e sim é proporcional ao nível de tensão do estímulo elétrico, de tal forma que, até certo limite, quanto maior for a voltagem do estímulo elétrico, mais fibras musculares serão capturadas e maior trabalho mecânico será desenvolvido.

Os efeitos sobre a performance cardíaca traduzem-se pelo aumento da função ventricular, que possivelmente ocorre por transferência de energia propulsora do músculo grande dorsal, no caso. Isso contribui para a diminuição da tensão da parede ventricular, aliviando o ventrículo e, conseqüentemente, mantendo-o dentro dos limites da sua reserva contrátil. A dilatação ventricular pode ser evitada pelo limite elástico do músculo grande dorsal, sem que haja restrição da complacência ventricular, evitando assim o aparecimento de arritmias e dilatações anulares das valvas mitral e tricúspide. A contração do músculo, de forma equaânime e sincrônica sobre a parede ventricular dilatada, certamente proporciona o aumento da função ventricular que é dependente das condições prévias do coração e da doença de base, sem sucesso em pacientes com miocardiopatia em fase muito adiantada, associada a grandes dilatações e a regurgitação mitral severa $4,7,33,34,36,40$.

\section{Músculo esquelético como suporte cardíaco}

Clinicamente, o músculo grande dorsal, além de ser utilizado em cardiomiopatias dilatadas, tem sido empregado também na reconstrução de ventrículos após excisão de tumores cardíacos ou aneurismas ventriculares e no auxílio de ventrículos discinéticos $4,7,8,11,12,20$

A estimulação elétrica crônica produz efeitos diferentes dos observados por estresse físico, visto que são acionadas diferentes vias de ativação 38 . É importante que a estimulação seja estável. O estresse físico consiste de impulsos intermitentes sobre o músculo, além disso, há influência seletiva sobre as unidades motoras $e$, conseqüentemente, alteração no efeito potencial de fatores desencadeados pela carga física. Por outro lado, características do músculo esquelético, altamente estáveis, sob o estresse físico, podem ser modificadas e mantidas por estimulação elétrica após longos períodos de estimulação ${ }^{15}$. Desse modo, a estimulação elétrica crônica do músculo esquelético permite o reconhecimento de todas as possibilidades adaptativas desse tecido muscular, que não seriam reveladas sob o estresse físico, responsável por alterações menos radicais.

Tanto o músculo esquelético como o cardiaco podem converter energia armazenada em trabalho mecânico. Ambos apresentam estrutura semelhante representada por miofibrilas com faixas e elementos longitudinais, alternados por bandas transversais claras e escuras, e um sistema de membrana extenso. Por outro lado, esses músculos diferem no número de mitocôndrias em cada célula, correspondente a $55 \%$ do volume celular no músculo cardíaco e apenas a $2 \%$ a $5 \%$ no músculo esquelético. Além disso, a célula cardíaca distingue-se da esquelética pela presença de um disco intercalar, que responde a um limiar baixo permitindo um rápido fluxo elétrico. O músculo esquelético, por sua vez, acentua ou reduz o estresse através da mobilização de um número maior ou menor de unidades motoras, isto é, por grupos de fibras musculares inervadas separadamente 15 .

Durante os últimos 50 anos, estudos histológicos e enzimáticos permitiram dividir as fibras musculares em 2 tipos. As fibras tipo I são semelhantes às fibras cardíacas em vários aspectos: apresentam metabolismo principalmente aeróbico, têm muitas mitocôndrias, contêm proteínas contráteis lentas como a miosina, com períodos longos de contração e são resistentes à fadiga. As fibras do tipo II de contração rápida dependem do metabolismo glicolitico, apresentam número reduzido de mitocôndrias e são expostas à fadiga rapidamente. O músculo esquelético é um tecido heterogêneo em que se observam esses dois tipos de fibras em proporções variáveis.

$\mathrm{Na}$ década de 70 , inúmeros estudos experimentais demonstraram que o músculo esquelético, submetido à estimulação crônica por dispositivos implantados ou por sistemas com eletrodos externos, aumenta sua resistência à fadiga com transformação de suas fibras ${ }^{21-24}$. A plasticidade do músculo esquelético, permitindo a transformação de suas propriedades fisiológicas e biológicas, foi revelada por BULLER et alii ${ }^{5,6}$ já no início da década de 60 , quando fizeram a reinervação cruzada entre nervos motores de músculos com contração rápida e lenta. Nesse caso, a transformação foi explicada por fatores quimiotróficos transmitidos ao longo do nervo motor ao músculo. Ainda nos anos 60 e 70, foi demonstrado por SALMONS 42, SALMONS \& VRBOVA ${ }^{45}$ e SRETER et alii 47 , que o nervo de músculo esquelético, submetido à estimulação com freqüência baixa através de um estimulador externo, resulta também na transformação das fibras. Posteriormente, o emprego desse músculo transformado para assistência cardíaca foi proposto pelo grupo de Stephenson, da Pensilvania, quando demonstram que estímulos de 2, 10 ou $35 \mathrm{~Hz}$ por 6 
BRAILE, D. M.; SOARES, M. J.F.; SOUZA, D. R. S.; SCHALDACH, M. - Cardiomioplastia: novo gerador da Biotronic. Rev. Bras. Cir. Cardiovasc., 9(1): 40-53, 1994.

a 8 semanas tornam o músculo predominantemente de contração lenta, com fibras aeróbicas do tipo I, altamente resistentes à fadiga 28,29 .

A transformação do músculo esquelético, refletindo sua adaptação mecânica e fisiológica, é revelada por alterações do retículo sarcoplasmático e mudanças no metabolismo enzimático com o aumento do ciclo oxidativo e redução da atividade glicolítica, com conseqüente transformação das fibras musculares glicolíticas de contração rápida em fibras de contração lenta, oxidativas e resistentes à fadiga. Com o estabelecimento da transformação, a densidade capilar acentua-se à medida que o metabolismo torna-se mais aeróbico e as cadeias pesada e leve da miosina também são modificadas. Isso evidencia a participação efetiva da estimulação elétrica na modificação da informação genética relativa à formação de proteínas contráteis adaptadas, nesse caso, a uma nova função 28,45 . As alterações nas isoformas da miosina e na cinética do transporte de cálcio são responsáveis pela redução na velocidade contrátil das fibras 45 .

Com a estimulação elétrica cria-se, portanto, um tipo de músculo que é definido não com a estimulação inicial, mas pela ativação elétrica crônica desse músculo, que irá desempenhar uma ação semelhante à cardíaca por longo tempo. Esse procedimento permite que todas as fibras participem do processo, com mudanças sincrônicas produzindo uma transformação completa na estrutura das miofibrilas em um período de 10 a 20 semanas.

Entretanto, causam preocupação os possíveis efeitos crônicos, aos quais estaria exposto o músculo esquelético submetido a um trem de impulsos elétricos necessários a sua ativação para desempenhar função semelhante. Isso é justificado pelo fato de que as contrações rigorosas causadas pelos impulsos elevam o consumo de $\mathrm{O}_{2}$, podendo resultar em isquemia, que certamente apresenta um papel importante na fadiga muscular 48,49 . É compreensível que, durante a dissecção do músculo esquelético, a circulação colateral fica comprometida, podendo levar à isquemia relativa. Por outro lado, sua estimulação imediata pode também danificá-lo 36 . Desse modo, para que o músculo esquelético possa ser usado para suportar pressão sistêmica, estimulado por um trem de impulsos elétricos, é necessário aguardar o desenvolvimento da circulação colateral, evitando a isquemia.

No final da década de 60 , HUME ${ }^{23}$ constatou uma redução da área da fibra de $30 \%$ a $40 \%$ após 6 semanas de estimulação do músculo esquelético, com freqüência de $10 \mathrm{~Hz}$. Entretanto, análises histoquímicas e bioquímicas revelaram um músculo com propriedades similares à fibra tipo I, sem sinais de lesão muscular com equilíbrio estável entre volu- mes de fibras e tecido conectivo intracelular. Isso apoia a idéia de que o músculo assim treinado pode ser usado para substituir o miocárdio por um longo período ${ }^{31}$.

\section{Estimulação elétrica do músculo esquelético 22}

As primeiras tentativas de assistência ventricular com músculo esquelético estimulado eletricamente foram realizadas com músculos submetidos a estimuladores convencionais, isto é, com pulsos únicos, com efeito hemodinâmico reduzido. O problema fundamental de um enxerto muscular, submetido a um estímulo elétrico sobre a parede do ventrículo esquerdo, consiste na divergência eletromecânica do músculo esquelético estriado e o sincício do miocárdio. A onda de despolarização do músculo esquelético, de $25 \mathrm{~ms}$ e a duração de sua contração de $100 \mathrm{~ms}$, são ambas muito menores do que a onda de despolarização do miocárdio e a duração de sua contração com 300 ms e 350 ms, respectivamente ${ }^{16}$. Isso significa que, sem um tipo de estímulo especial, o músculo esquelético tem um período de contração muito curto em relação à contração cardíaca (Figura 1).

Devido à ineficiência, como suporte para a cardiomioplastia, dos estimuladores convencionais e de estimuladores tetânicos, que possibilitam o aumento da força e duração da contração, mas com fadiga precoce inevitável, diversos modelos de estimuladores de pulso foram desenvolvidos, com destaque para o de pulso múltiplo, em que se associou um marcapasso padrão e um modulador de freqüência 17, 26. Esse tipo de estimulador, apoiado pelo princípio da somatória das contrações do músculo esquelético, possibilita aumentar o trabalho muscular comparado aos padrões de estimulação convencional, responsáveis por contrações simples.

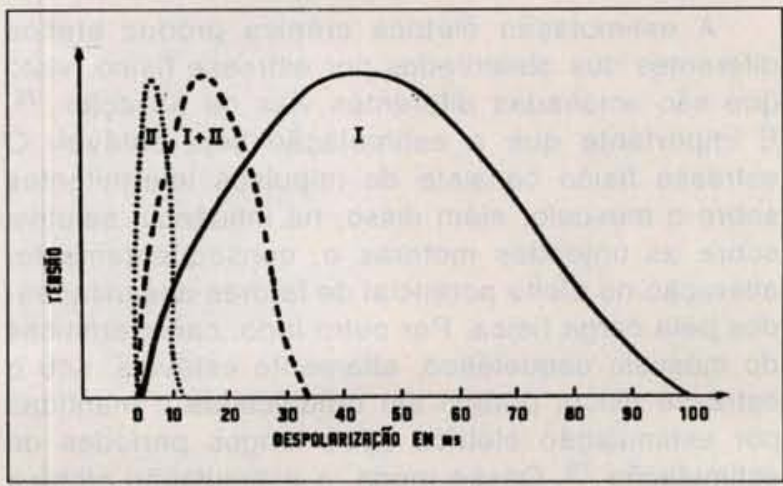

Fig. 1 - Curvas ilustrando a duração da contração muscular de acordo com os tipos de fibra: fibras tipo I; fibras tipo II; fibras tipos I e II (músculo transformado) (modificado de Grandjean 22 
BRAILE, D. M.; SOARES, M. J. F.; SOUZA, D. R. S.; SCHALDACH, M. - Cardiomioplastia: novo gerador da Biotronic. Rev. Bras. Cir. Cardiovasc., 9(1): 40-53, 1994.

Um dos problemas principais que interferem no sucesso da substituição do músculo cardíaco é a estimulação sincrônica do miocárdio e o músculo esquelético ${ }^{21}$. A estimulação desse músculo a longo prazo tem sido possível graças a eletrodos especiais associados à estimulação progressiva seqüencial, adaptando-o à função cardiaca. Estudos têm mostrado que o músculo grande dorsal, submetido a um programa específico de estimulação, é capaz de produzir contrações adequadas através do aumento da resistência ao estresse físico, decorrente da transformação gradual de fibras glicolíticas expostas à fadiga em oxidativas lentas altamente resistentes 20 .

A resposta da fibra muscular à estimulação elétrica pode ser beneficiada pelos parâmetros: elétrico, local e posição do eletrodo e diâmetro da fibra nervosa. O primeiro deles destaca que a força muscular gerada pode ser controlada pela variação da amplitude ou largura de pulso. O aumento de uma ou de outra será suficiente para estimular fibras pequenas como também as fibras mais distantes, através da polarização das membranas. Nesse caso, nenhuma resposta muscular é observada até que o limiar da maioria das unidades expostas à estimulação for alcançada. Por outro lado, o aumento acima dos níveis necessários não resulta em aumento adicional da força. A freqüência de estimulação mantida durante a contração determina a fadiga do músculo, que depende também do tipo de fibra muscular, seu condicionamento e o ciclo de trabalho ao qual é submetida, representado por tempo de estimulação versus tempo de descanso. Com a redução da freqüência de estimulação minimiza-se a fadiga.

Um outro parâmetro importante a ser considerado é o local e posição do eletrodo no músculo. O nervo, por apresentar limiar de estimulação mais baixo do que as fibras musculares, pode parecer a área mais apropriada para o implante do eletrodo, minimizando o problema de fibrose na área do eletrodo, além de aglomerar eficientemente as fibras musculares. Entretanto, enxertos pediculados dispostos sobre a parede ventricular, submetidos à estimulação pela contração do pedículo estimulado, podem desencadear disrritmias e redução do rendimento cardíaco 26 . Por outro lado, eletrodos implantados diretamente no músculo parece a opção correta para enxertos de uso crônico, sem problemas graves de fibrose ${ }^{9}$. A estimulação elétrica das fibras musculares esqueléticas é significativamente mais baixa do que a dos nervos motores, ou seja, as fibras apresentam um limiar mais alto. O estímulo direto da fibra muscular com pulsos de curta duração necessita de uma amplitude maior do que o estímulo indireto do músculo, isto é, dos nervos motores. Com o aumento da largura de pulso, reduz-se sua amplitude. Entretanto, a energia alta pode comprometer a estabilidade eletroquímica do eletrodo e ser prejudicial aos tecidos neurais. Desse modo, a distância do eletrodo ao nervo é um parâmetro importante na determinação do limiar da amplitude de pulso. Assim sendo, no músculo desnervado pela ausência de difusão das unidades motoras, cada fibra muscular necessita ser despolarizada individualmente, o que pode ser muito difícil em grandes músculos.

Finalmente, o diâmetro da fibra nervosa, também importante na estimulação elétrica da fibra muscular, evidencia que as fibras grandes apresentam limiar de estimulação inferior ao das fibras pequenas, devido às suas grandes distâncias internodais.

\section{Programa de estimulação do músculo grande dorsal}

O protocolo de estimulação do músculo grande dorsal, conduzido de maneira seqüencial e progressivo no pós-operatório de pacientes submetidos à cardiomioplastia em nosso Serviço, tem como referência o programa de estimulação desenvolvido por CARPENTIER \& CHACHQUES ${ }^{8}$.

A cardiomioplastia, realizada sem o auxílio de circulação extracorpórea, é desenvolvida em dois tempos, iniciando-se com a dissecção do músculo grande dorsal, seguida de esternotomia mediana para acesso ao coração. A fase seguinte, ou seja, a estimulação do músculo grande dorsal é decisiva na cardiomioplastia, determinando o seu sucesso como suporte cardíaco. É registrado abaixo o programa de estimulação do músculo grande dorsal desenvolvido em nosso Serviço.

1 a 2 semanas após a cardiomioplastia: período sem estimulação. Essa fase é necessária para a formação de circulação colateral e aderências entre o músculo e o miocárdio, além da regressão do edema e maturação do enxerto.

3 a 4 semanas: a eletroestimulação inicia-se com pulsos únicos na razão $2: 1$, em perfeito sincronismo com a abertura da valva aórtica.

5 a 6 semanas: o número de espículas é aumentado para dois, com estimulação na razão 2:1.

7 a 8 semanas: aumento do número de espículas para três, com estimulação na razão 2:1 (Figura 2).

O número ideal de espículas ao completar dois meses de cirurgia, culmina com um trem de até 6 a 8 pulsos, com freqüência de $30 \mathrm{~Hz}$, de acordo com os parâmetros avaliados pela ecocardiografia. Recentemente temos verificado que, sincronizando a contração do grande dorsal com a abertura da valva aórtica, bastam 3 ou 4 espículas com freqüência de $30 \mathrm{~Hz}$ para obter-se uma boa contração 


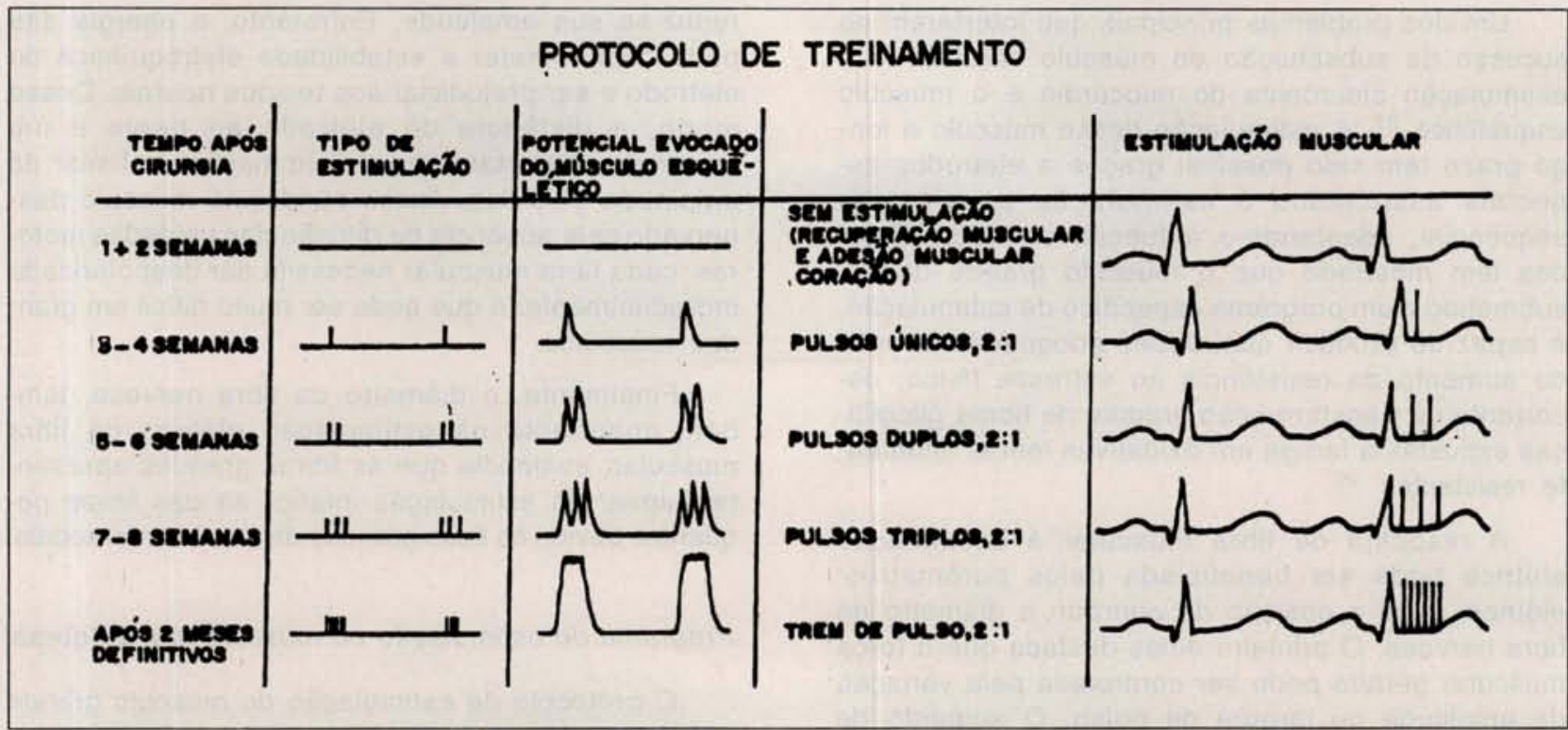

Fig. 2 - Protocolo de estimulação no pós-operatório. O músculo grande dorsal é elétricamente condicionado durante um periodo de 6 semanas, geralmente a partir do $14^{2}$ dia do pós-operatório. Após o início do protocolo, a estimulação é contínua.

muscular, evitando tanto a fadiga muscular como também alguma interferência do músculo ainda contraído com a diástole cardíaca.

Para estimulação elétrica do músculo grande dorsal, é utilizado em nosso Serviço o gerador de pulsos "Myos" (Biotronik ${ }^{R}$ ) (Figura 3 ), em sincronização com o miocárdio (Figura 4). Esse sistema funciona com três eletrodos, dois deles são destinados à estimulação do músculo esquelético e o outro, normalmente epimiocárdico, é usado para detecção de sinais próprios do coração que comandam a estimulação do músculo esquelético. Quando necessário, eletrodo cardíaco pode também estimular o miocárdio exercendo, nesse caso, a função de marcapasso.

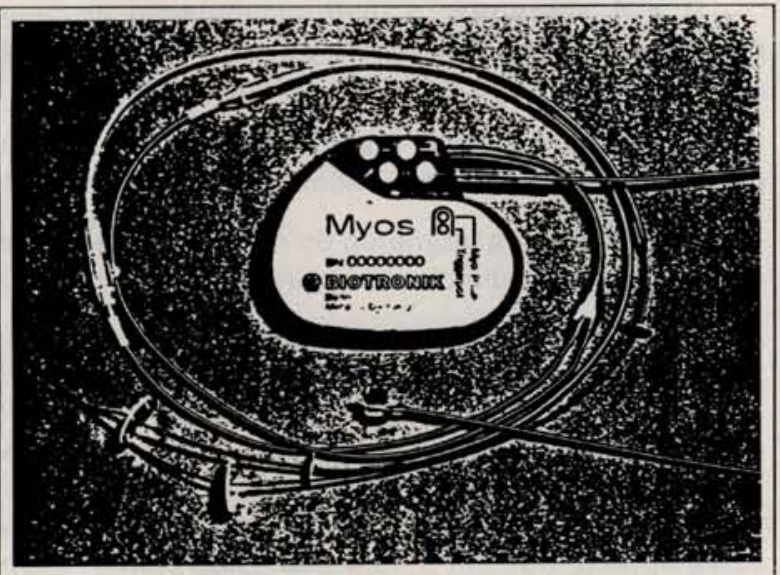

Fig. 3- Cardiomioestimulador Myos e eletrodos Biotronik R.

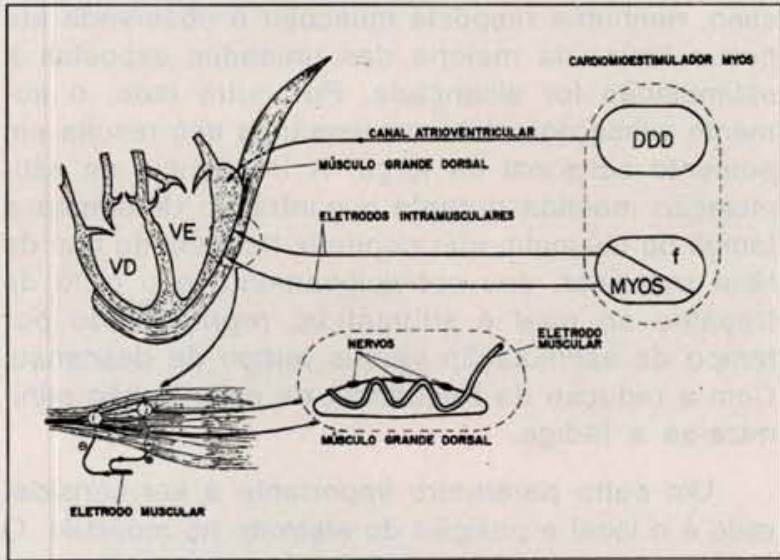

Fig. 4 - Esquema demonstrativo do funcionamento do cardiomioestimulador Myos (Biotronik)

Esse tipo de cardiomioestimulador com o circuito eletrônico e uma bateria de lítio, apresenta uma carcaça de titânio que o sela hermeticamente. No circuito eletrônico é armazenado o programa de estimulação responsável pelos diferentes modos operacionais. A adaptação não invasiva do modo operacional é realizada por um programa de computação.

A programação do gerador, identificado como um marcapasso de dupla câmara, é feita por computador PMS600 ou PMS1000 (Biotronik), com um cabeçote de programação adaptado de tal forma que permite a comunicação entre o Myos e o programador. Assim sendo, é possível uma seleção rápida e fácil do programa desejado e de sua trans- 
BRAILE, D. M.; SOARES, M. J. F.; SOUZA, D. R. S.; SCHALDACH, M. - Cardiomioplastia: novo gerador da Biotronic. Rev. Bras. Cir. Cardiovasc., 9(1): 40-53, 1994.

missão ao cardiomiestimulador. Para documentação pode ser adaptada uma impressora.

A estimulação caracteriza-se pela sincronização da contração muscular com a sístole ventricular, considerando-se a abertura da valva aórtica e os tempos de abertura e fechamento da valva pulmonar. O pulso ventricular é liberado após o retardo atrioventricular, gatilhando um trem de pulso para estimular o músculo esquelético. O número de espículas e a proporção entre a freqüência do miocárdio e a freqüência da liberação do trem de pulso são ajustados através da colocação de um imã, de acordo com procedimento descrito a seguir:

1 Seleção dos valores dos parâmetros desejados para o marcapasso de dupla câmara.

1.1 Seleção do modo DDDc (ou DOO), amplitude ventricular de 4,8 volts, largura de pulso ventricular de $0,5 \mathrm{~ms}$ e período refratário ventricular de $300 \mathrm{~ms}$. Esses valores são indispensáveis para o funcionamento do circuito do trem de pulso e não devem ser alterados.

1.2 Seleção do intervalo de sincronização entre o evento miocárdico (sentido ou estimulado) e 0 início do trem de pulso, ajustando o retardo atrioventricular de sensibilidade e de estimulação. O intervalo de sincronização resultante será o nominal mais $30 \mathrm{~ms}$.

2 O marcapasso é ligado e desligado por breve passagem do imã sobre a região de implante.

3 Seleção do número de espículas por trem de pulso e do sistema de redução da freqüência de estimulação.

3.1 Posicionamento do imã sobre o local de implante do gerador de pulso. O tempo de permanência do imã sobre a região implantada define o programa de mioestimulação no estimulador. O número de pulsos e a proporção dos batimentos podem ser ajustados. Atingida a programação desejada, o imã é retirado. O estimulador continuará então a estimular com este programa.

O retardo AV é de $100 \mathrm{~ms}$ durante a colocação do imã, independente dos valores selecionados no programa do marcapasso de dupla câmara. A cada dois intervalos básicos acrescenta-se uma espícula no trem de pulso até no máximo oito. Depois disso a freqüência de acoplamento é modificada de 1:1 para 2:1, por exemplo, um trem de pulso é liberado a cada dois batimentos cardíacos (sentido e estimulado). O número de pulsos no trem de pulso é reduzido automaticamente para um. Com o imã sobre - marcapasso o número de pulsos por trem de pulso aumenta novamente, podendo chegar até a oito pulsos. A seguir é alterada a freqüência de acomplamento de 2:1 para $3: 1$. Assim, o trem de
TABELA 1

FREQÜÊNCIA MIOCÁRDICA EM BATIMENTOS POR MINUTO (BPM) E SEUS RESPECTIVOS NÚMEROS DE PULSOS

\begin{tabular}{cc}
\hline FREQÜENCIA (BPM) & NoDE PULSOS \\
\hline$<65$ & 1 \\
$65-74$ & 2 \\
$75-84$ & 3 \\
$85-94$ & 4 \\
$95-114$ & 5 \\
$>115$ & 6 \\
\hline
\end{tabular}

pulso só é liberado após o terceiro evento miocárdico (sentido ou estimulado). O número de espículas de pulso é reduzido novamente a um. Isso continua até que o estado inicial seja alcançado e o procedimento se repita.

Para facilitar a programação, interrompe-se o procedimento de tempos em tempos através da remoção do imã e verificação do progresso no estabelecimento dos parâmetros de mioestimulação. Aplicando-se o imã novamente, inicia-se o sistema no ponto em que foi parado.

A redução do pulso indica a relação entre QRS/ pulso, ou seja, pode ser programada de modo que o evento sentido ou estimulado no ventrículo corresponda a um pulso, ou uma relação menor entre QRS/pulso.

Pode ser relacionada também uma redução automática entre eventos cardíacos e pulso, de forma que o pulso não ultrapasse uma freqüência de 30 pulsos por minuto. A redução de pulso pode ser representada por: $1: 1,2: 1,3: 1,4: 1$.

Um novo modelo Myos 03, já em avaliação, permite toda a programação por meio de um "software".

\section{Eletrodo de implantação muscular}

Para a utilização do Myos foi desenvolvido um

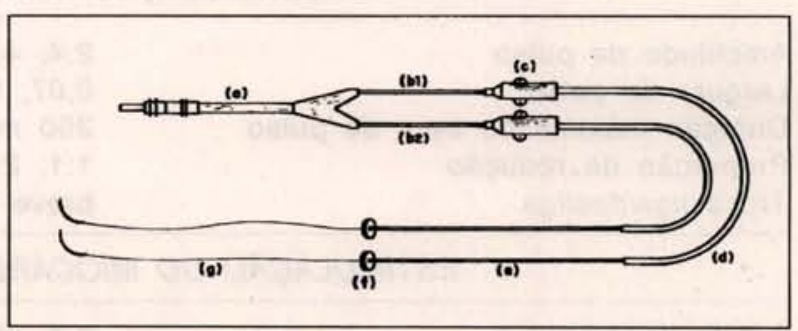

Fig. 5 - Esquema do eletrodo de implantação muscular modelo CM60BP (Biotronik). (a) Conexão do eletrodo com o mioestimulador. (b1) Catódio. (b2) Anódio. (c) Fixadores da capa móvel de silicone. (d) Capa móvel de silicone. (e) Eletrodo. (f) Discos de silicone. (g) Fio de Polipropileno 3.0 com agulha. 
BRAILE, D. M.; SOARES, M. J. F.; SOUZA, D. R. S.; SCHALDACH, M. - Cardiomioplastia: novo gerador da Biotroǹic. Rev. Bras. Cir. Cardiovasc., 9(1): 40-53, 1994.

eletrodo implantável especial “CM 60 - BP” (Biotronik) (Figuras 4 e 5). Esse tipo de eletrodo permite uma grande área de contato quando instalado no músculo, em especial na região dos nervos motores. Sua estrutura fractal permite amplo contato do eletrodo com as células musculares e nervosas. As pontas do eletrodo são de iridium espiralado, sem isolamento, permitindo ajuste após o seu implante no músculo através de capa móvel de silicone, que possibilita uma variação de comprimento ativo. Esse eletrodo é inserido no músculo esquelético, de maneira que estimula diretamente seus nervos motores, garantindo que a estimulação seja efetiva e com economia de energia. Existem vários eletrodos destinados à detecção da atividade elétrica do coração e estimulação do miocárdio. Entretanto, o de tipo DM 50-UP (Biotronik) (Figura 6) de superfície microporosa garante níveis adequados dessas atividades. A estrutura fractal permite uma íntima interação entre os eletrodos e as células "ouvindoas" ou estimulando-as em níveis de baixa energia.

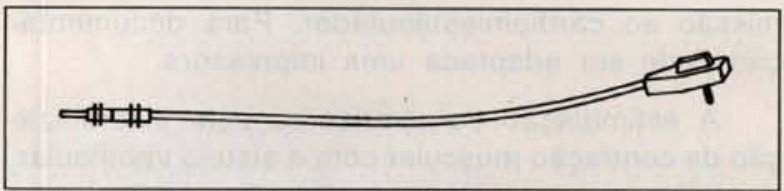

Fig. 6- Esquema do eletrodo epicárdico de tipo DM-50-UP (Biotronik).

A conexão do eletrodo é do tipo IS-1 bipolar, em que o anodo (pólo positivo) e o catodo (pólo negativo) são identificados para facilidade da implantação. Nos terminais distais dos pólos existe um fio de sutura com agulha curva, que permite a fixação do eletrodo ao músculo. Depois da fixação o fio e a agulha são cortados e retirados. A porção distal do eletrodo CM-60 BP, formada pelas espirais metálicas, deve ser mantida. Após fixação da área de contato do eletrodo no músculo, deve ser fixada a porção isolada, através dos pontos de fixação existentes na borracha de silicone, que acompanha - eletrodo, utilizando-se um fio de sutura não reabsorvivel.

\section{CARACTERISTICA DO MYOS}

Estimulação

Estimulação, detecção Unipolar (miocárdio)

Bipolar (músculo esquelético)

PULSO DO GERADOR E PARÂMETRO CONTROLE

Modo ${ }^{1}$

Freqüência básica

Histerese

Freqüência máxima

Dupla demanda
DDDC, DOO, SSI, SOO

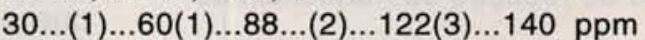

$30 \ldots$ (1)...99...(2)...122...(3)...140 ppm

$100,110,120,130,140,160,185$ ppm

ligado/desligado

\section{SINCRONIZAÇÃO DOS INTERVALOS}

Intervalo de Estimulação do miocárdio ${ }^{2}$ Intervalo de sensibilidade do miocárdio3 Tempo de sensibilidade para freqüências dinâmica (individual)
$15,50,75,100,120 \ldots(10) \ldots 200,225,250,300 \mathrm{~ms}(+-30 \mathrm{~ms})$

$15,50,75,100,120 \ldots(10) \ldots 200,225,250,300 \mathrm{~ms}(+-30 \mathrm{~ms})$.

$<70,70-90,91-110,111-130,>130 \mathrm{bpm}$

\section{ESTIMULAÇĀO DO MÚSCULO ESQUELÉTICO}

\section{Amplitude de pulso}

Largura de pulso

Duração máxima do trem de pulso

Proporção de redução

Troca liga/desliga
2,$4 ; 4,8 \mathrm{~V}$

0,$07 ; 0,11 ; 0,15 ; 0,35 \mathrm{~ms}$

$250 \mathrm{~ms}$

$1: 1 ; 2: 1 ; 3: 1 ; 4: 1$ (ajustável por imã)

breve toque com imã

ESTIMULAÇÃO DO MIOCÁRDIO (FUNÇÃO DO MARCAPASSO)

Amplitude de pulso

Largura de pulso

Sensibilidade

Periodo refratário
$0,1 \ldots(0,1) \ldots 4,8 \ldots(1,2) \ldots 9,6 \quad \mathrm{~V}$

$0,25,0,50,0,75,1,0 \mathrm{~ms}$

$0,5 \ldots(0,5) \ldots 1,5 \ldots(0,5) \ldots 7,5 \mathrm{~V}$

$200 \ldots(25) \ldots 400 \ldots(25) \ldots 775 \mathrm{~ms}$ 


\section{Diretrizes para definição do programa de estimulação}

As diretrizes para a programação mais adequada do cardiomioestimulador Myos (Biotronik) em pacientes submetidos à cardiomioplastia são referidas a seguir:

1 As funções do marcapasso de dupla câmara, que faz parte do sistema do Myos, são programadas de maneira habitual de acordo com os conhecimentos gerais sobre terapia com marcapasso.

2 A ecocardiografia, com recursos de imagens, congelamento e medidas, é indispensável para - sucesso da programação. Nesse caso, possibilita o ajuste de sincronismo da assistência biomecânica circulatória.

3 Para a programação do cardiomioestimulador, o momento da sincronização do trem de pulso com a abertura da valva aórtica é de extrema importância. $O$ modo $M$ de alta velocidade é utilizado para avaliar este sincronismo.

4 Para o paciente esta otimização do programa pode determinar um importante aumento do débito cardíaco. Os valores variam de 200 a $1000 \mathrm{ml} / \mathrm{min}$.

5 Em nosso Serviço é utilizado com sucesso o instante da abertura da valva aórtica após o fechamento da valva mitral como referência para o sincronismo do trem de pulso. Desse modo, a força mecânica do enxerto muscular é concentrada no momento mais efetivo do encurtamento ventricular, que é o $1^{\circ}$ e $2^{\circ} 1 / 4$ da fase da é sítole cardíaca (Figura 7).

6 O número ideal de pulsos no trem de pulso é determinado pelo intervalo de tempo (em $\mathrm{ms}$ ) durante a abertura da valva aórtica e o fechamento da valva pulmonar. Este intervalo é mensurado através do ecocardiograma. A valva aórtica é visibilizada através do modo $M$ após um perfeito sincronismo muda-se o transdutor para a valva pulmonar, sendo medido o intervalo de tempo para o seu fechamento. O fechamento da valva pulmonar é utilizado como referência, pois é neste momento que o ciclo

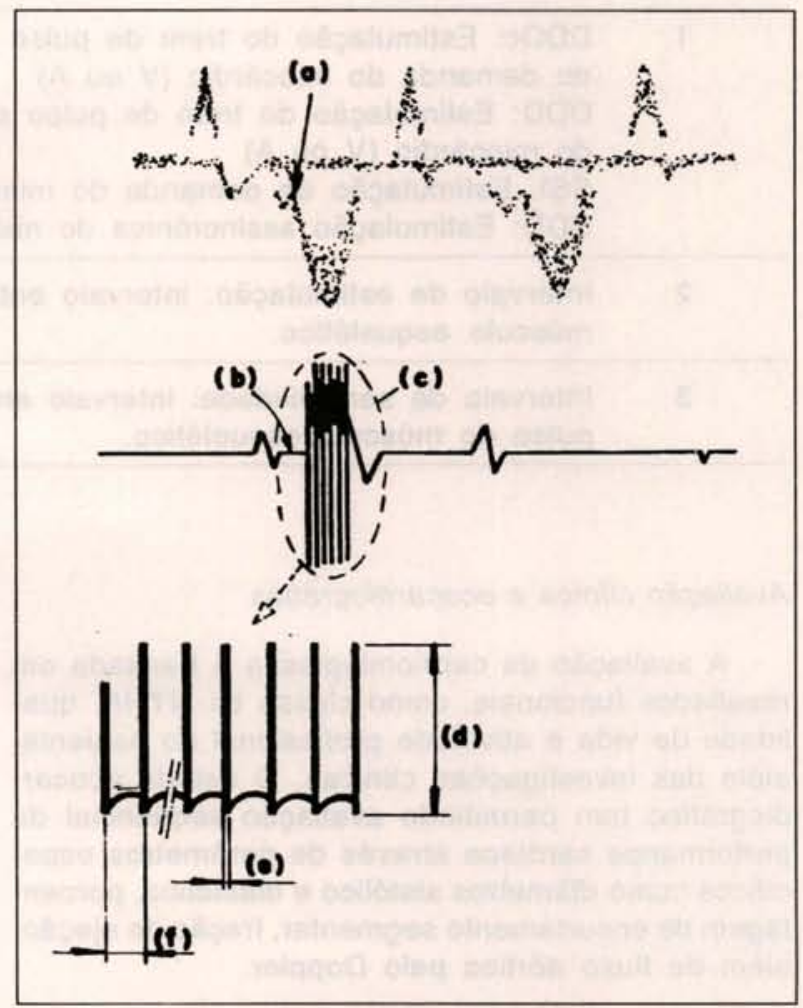

Fig. 7 - Esquema do sincronismo da estimulaçăo muscular com a abertura da valva aórtica. (a) Abertura da valva aórtica. (b) Atraso da estimulaçāo sincronizada com a abertura da valva aórtica. (c) Estimulaçăo elétrica do músculo grande dorsal. (d) Amplitude de pulso. (e) Largura de pulso. (f) Intervalo de pulso.

cardíaco se encontra na protodiástole, com pouca quantidade de sangue, sendo mandada para a circulação. Nesse caso, não há necessidade de se manter a estimulação com conseqüente contração do músculo esquelético, pois será inefetiva.

A energia elétrica de estimulação e a energia muscular podem ser poupadas pela determinação prévia do intervalo de tempo e pelo cálculo do número de trens de pulso. O intervalo entre 2 pulsos é de $30 \mathrm{~ms}$, logo, dividindo-se o intervalo de tempo por 30 determina-se de maneira otimizada o número de pulsos do trem de pulso.
Capacidade nominal da bateria

Circuito

Carcaça

Conexão dos eletrodos

Dimensões

Peso
2,55 AL, Li/l

Eletrônicos híbridos LSI-CMOS

Titânio

$1 \times 1$ S- 1 bipolar, $1 \times 1$ S- 1 unipolar

$66 \mathrm{~mm} \times 52 \mathrm{~mm} \times 10 \mathrm{~mm}$

$49 \mathrm{~g}$ 
BRAILE, D. M.; SOARES, M. J. F.; SOUZA, D. R. S.; SCHALDACH, M. - Cardiomioplastia: novo gerador da Biotronic. Rev. Bras. Cir. Cardiovasc., 9(1): 40-53, 1994.

1 DDDc: Estimulação do trem de pulso do músculo esquelético, estimulação

de demanda do miocárdio ( $V$ ou $A$ )

DDD: Estimulação do trem de pulso do músculo esquelético, estimulação assincrônica do miocárdio ( $V$ ou $A$ )

SSI: Estimulação de demanda do miocárdio ( $V$ ou $A$ )

DDD: Estimulação assincrônica do miocárdio ( $V$ ou $A$ )

2 Intervalo de estimulação: intervalo entre estimulação miocárdica e o trem de pulso do músculo esquelético.

3 Intervalo de sensibilidade: intervalo entre a sensibilidade do evento elétrico e o trem de pulso do músculo esquelético.

\section{Avaliação clínica e ecocardiográfica}

A avaliação da cardiomioplastia é baseada em resultados funcionais, como classe da NYHA, qualidade de vida e atividade profissional do paciente, além das investigações clínicas. $O$ estudo ecocardiográfico tem permitindo avaliação seqũencial da performance cardiaca através de parâmetros específicos como diâmetros sistólico e diastólico, porcentagem de encurtamento segmentar, fração de ejeção, além de fluxo aórtico pelo Doppler.

A avaliação clínica da cardiomioplastia tem como base os resultados obtidos de 32 pacientes submetidos a essa operação sob nossa orientação, que apresentavam alto risco de falecerem após o reconhecimento da disfunção miocárdica grave. Esses pacientes apresentavam idade de 22 a 72 anos (média $=46,2$ anos), sendo 9 do sexo feminino e 23 do sexo masculino.

A maioria $(69 \%)$ dos pacientes tinha miocardiopatia dilatada por causa indeterminada, $25 \%$ de origem chagásica, $3,0 \%$ virótica e $3,0 \%$ por periparto.

\section{Pré-operatório}

$\mathrm{Na}$ avaliação pré-operatória detectou-se que 21 pacientes estavam em classe funcional IV e $11 \mathrm{em}$ classe III da NYHA. Os pacientes em classe funcional IV, com fração de ejeção abaixo de $30 \%$ pela ecocardiografia, foram submetidos a tratamento clínico intensivo, de modo que à época da operação encontraram-se em melhor estado funcional (III e II).

Os pacientes foram submetidos a: radiografia de tórax para avaliação da área cardíaca e dos pulmões; tomografia computadorizada do tórax, quando necessário, para descartar doenças pulmonares ou pleurais; testes funcionais pulmonares; eletrocardiograma simples e dinâmico para exclusão de arritmias muito graves que pudessem contra-indicar o procedimento; cineangiocoronariografia para avaliação da morfologia e estado funcional dos ventrículos, assim como as condições das artérias coronárias e valvas cardiacas; ecocardiograma para avaliação das valvas atrioventriculares e semilunares dos diâmetros dos ventrículos direito e esquerdo, bem como a função cardíaca através da fração de ejeção, encurtamento segmentar e débito cardíaco; além disso receberam a atenção de psicólogo e assistente social.

\section{Pós-Operatório}

Dos 32 pacientes submetidos à cirurgia, 12,5\% do total e $3,1 \%$, excluindo-se os chagásicos foram a óbito antes de completarem 1 mês de pós-operatório. As causas dos óbitos foram: embolia pulmonar maciça em um paciente chagásico e fibrilação ventricular em outros dois, além de um de etiologia idiopática. Índices aceitáveis comparados à mortalidade de até $60 \%{ }^{16}$ para tratamentos clínicos e de $12,8 \%$ a $18,2 \%$ de mortalidade precoce registrados por outros Serviços $16,44-46$. Na verdade, a mortalidade operatória apresenta-se intimamente relacionada ao estado pré-operatório do paciente, podendo ser controlada com a seleção dos pacientes ${ }^{14,47}$.

O tempo de seguimento dos 28 pacientes que deixaram o hospital totalizou 454 meses, com média de 14,2 meses. O índice de mortalidade tardia foi de $3,6 \%$, retirando-se os chagásicos, e de $14,3 \%$ considerando o total de pacientes operados. Os óbitos foram referentes a 3 pacientes chagásicos que faleceram por insuficiência cardiaca congestiva (ICC), 2 deles, e fibrilação ventricular (FV), apenas 1 , após 4,12 e 21 meses, respectivamente. Entre os demais pacientes, $1 \mathrm{com}$ miocardiopatia idiopática faleceu por ICC após seis meses de evolução. Esses resultados são comparáveis ou inferiores aos índices de $9,1 \%$ a $31,2 \%$ de mortalidade tardia referidos por outros autores 14, 44-46.

As complicações fatais foram responsáveis por índices de incidência linearizada de $7,9 \%$ de ICC/ paciente-ano e de $2,6 \%$ de FV/paciente-ano. $O$ índice 
total de sobrevida foi de $75,3+-0,22 \%$ após 6 anos e de $94,4+-0,1 \%$ após 5 anos, descartando-se os pacientes chagásicos. A literatura consultada revelou índice de sobrevida atuarial em torno de $65 \%$ em 24 meses de seguimento de pacientes com cardiomiopatias dilatadas, incluindo até $19 \%$ de chagásicos 24,33 , contra os $25 \%$ dos pacientes com essa etiologia, apresentados nesse estudo. Por outro lado, índice de $70,4 \%$ de sobrevida foi registrado por CARPENTIER et alii ${ }^{46} \mathrm{em} 84$ meses, em face de $20 \%$ a $30 \%$ para pacientes clínicos após 2 a 3 anos de seguimento 10,33 .

A classe funcional dos pacientes é preditiva no indice de sobrevida. Dos 24 pacientes em classes III e IV da NYHA no pré-operatório, excluindo-se os óbitos hospitalares e tardios, 17 (71\%) evoluíram para a classe I e $7(29 \%)$ para a classe II. O índice de mortalidade entre os pacientes em classe IV foi de $28,6 \%(6 / 21)$ em face de $18,2 \%$ (2/11) para aqueles em classe III, com a maioria dos óbitos no primeiro ano, justificando a indicação precoce da cardiomioplastia. Todos os pacientes mostraram-se tolerantes à estimulação crônica do músculo grande dorsal.

A cardiomioplastia foi efetiva na assistência do ventrículo esquerdo. O estudo comparativo entre os valores médios de pré e pós-operatórios, considerando o seguimento médio de 14,2 meses, evidenciou em nível de $\mathrm{p}<0,05$, redução do diâmetro diastólico de $72,7+-2,8 \mathrm{~mm}$ para $70,7+-1,4 \mathrm{~mm}$ e redução significativa do diâmetro sistólico de 61,2 +- $2,7 \mathrm{~m}$ para $55,1+-1,8 \mathrm{~mm}$, e elevação estatisticamente significativa do encurtamento segmentar de $15,9+-1,9 \%$ para $22,8+-1,2 \%$ e da fração de ejeção de $38,3+-3,7 \%$ para $51,0+-1,8 \%$. Esses índices são satisfatórios comparados aos da literatura, com elevação significativa do diâmetro sistólico de $23,6 \%$ para $37,7 \%$, do encurtamento segmentar de $12 \%$ para $16,4 \%$ e da fração de ejeção de $22,9 \%$ para $30,5 \%$, em 18 meses de acompanhamento 33 .

A elevação da fração de ejeção e do encurtamento segmentar, detectada já aos 3 meses da operação justifica-se principalmente pela ação da contração sincrônica do enxerto muscular sobre a superfície ventricular ${ }^{15}$. Isso reflete que, após esse período, o músculo grande dorsal encontra-se devidamente treinado.

O diâmetro ventricular diastólico determina a melhora da função do ventrículo esquerdo, detectada em pacientes com valores inferiores a $70 \mathrm{~mm}$ para esse parâmetro, enquanto que valores superiores a $75 \mathrm{~mm}$ apresentam resultados insatisfatórios ${ }^{10}$. Parece haver, ao longo do tempo, estabilização no processo de dilatação ventricular na maioria dos pacientes $4,10,24$.
A cardiomioplastia tem sido de grande importância para os pacientes com miocardiopatia dilatada, inclusive para os de etiologia chagásica, tendo em vista que o transplante cardíaco nesses pacientes tem resultado em alto índice de recorrência de infecção por $T$. cruzi ${ }^{18}$. Estudos anatomopatológicos de pacientes chagásicos demonstraram que o músculo esquelético mantém sua capacidade de adaptação à fadiga, com morfologia preservada, mesmo na presença de reagudização da miocardite chagásica 4,46 .

Os pacientes indicados para a cardiomioplastia, apesar da descompensação freqüente, devem ser passíveis de recuperação podendo, com o tratamento adequado, ser revertidos para um estado clínico relativamente estável. Tendo em vista que esses pacientes expostos a um trauma cirúrgico têm que suportar um período de pós-operatório imediato sem o auxílio muscular, a escolha do paciente parece ser a chave para o bom resultado operatório a curto e longo prazos.

Com base na experiência adquirida ao longo desse período, é possível definir o perfil do paciente indicado para a cardiomioplastia:

1 Pacientes abaixo de 70 anos em classe funcional IV que tenham sido revertidos para classe III ou II, de preferência.

2 Ausência de arritmias malignas e incontroláveis.

3 Diâmetro diastólico do ventrículo esquerdo pela ecocardiografia menor que $75 \mathrm{~mm}$.

4 Fração de ejeção do ventrículo esquerdo maior que $15 \%$.

5 Pacientes com cardiopatia fundamentalmente dilatada, evitando-se os hipertróficos.

6 O comprometimento do ventrículo direito (VD) é altamente preditivo quanto ao sucesso da operação. Há referência de que pacientes com comprometimento importante de VD apresentam um prognóstico pior que os pacientes com grave comprometimento de VE, evoluindo com alta taxa de mortalidade por ICC refratária e embolia pulmonar 43 .

7 A miocardiopatia deve estar controlada, sendo contra-indicada em casos de doenças agudas ou em evolução.

8 O paciente deve apresentar bom estado geral, com ausência de falência de outros órgãos. A sua musculatura esquelética deve estar preservada e livre de processos patológicos específicos.

9 A pressão do tronco pulmonar, assim como a resistência vascular pulmonar, devem estar dentro dos limites da normalidade.

10 O paciente não pode ser viciado em drogas ou álcool. 
11 O nível sócio-econômico e a residência do paciente devem ser considerados como fatores coadjuvantes na indicação.

\section{Futuro}

Considerando que o músculo esquelético desenvolve tensão suficiente para manter a circulação; considerando que o mesmo pode ser transformado em músculo resistente à fadiga, este campo encontrará grande aplicação no futuro, seja para reforçar ou substituir partes do coração ou para criação de um coração muscular.

Já dispomos de um novo cardiomioestimulador em que a informação autônoma do batimento cardíaco é usada para sincronizar o cardiomioestimulador usando as vantagens das informações cronotrópica e dromotrópica para reestabelecer o controle hemodinâmico do rendimento cardíaco. 0 número de espículas é proporcional à frequeência cardíaca.

Portanto, a otimização do sistema pode ser obtida controlando a força contrátil do músculo grande dorsal, pela modificação do retardo eletromecânico, ou duração do trem de pulso ou ainda a relação a QRS/estimulação muscular.

Um dos pontos em destaque é que o músculo esquelético submetido a um período de repouso torna-se capaz de um desempenho muito maior no auxílio circulatório do que quando em trabalho constante. As necessidades de débito cardiaco também variam de acordo com a atividade do individuo.
Assim, quando em repouso ou durante o sono, mesmo o cardiopata não necessita de grande auxílio para o seu fraco coração.

A possibilidade de avaliar-se o tônus simpático através de a) período de pré-ejeção (PEP) medido através da bioimpedância intracardíaca e b) da medida da resposta ventricular evocada (repolarização), que indica a velocidade do influxo de cálcio nas células e, portanto, o estado contrátil do miocárdio, permite-nos também conhecer a necessidade de maior ou menor auxílio por parte do coração.

Em conjunto com a Biotronik, estamos desenvolvendo um cardiomioestimulador que "sente" o tônus simpático do paciente e a necessidade de maior débito cardíaco. Com esta informação disponível será possível modular a estimulação do músculo esquelético de acordo com a demanda circulatória do paciente. Quando este estiver em repouso, o músculo será estimulado apenas com uma espícula, com voltagem mínima simplesmente para manter sua viabilidade. No momento de esforço ou estresse, o número e amplitude de espículas aumenta de forma proporcional ao tônus simpático, auxiliando de forma efetiva a circulação.

Teremos, assim, o músculo esquelético adaptado de forma fisiológica, a demanda do débito cardíaco mantendo-o em repouso quando possível e usando toda sua energia quando necessário. Desta maneira, a otimização do suporte ventricular poderá ser garantido, resultando em mínima fadiga e longa preservação do grande dorsal no suporte à circulação. 
BRAILE, D. M.; SOARES, M. J. F.; SOUZA, D. R. S.; SCHALDACH, M. - Cardiomioplastia: novo gerador da Biotronic. Rev. Bras. Cir. Cardiovasc., $9(1): 40-53,1994$.

RBCCV 44205-230

BRAILE, D. M.; SOARES, M. J. F.; SOUZA, D. R. S.; SCHALDACH, M. - Cardiomyoplasty: a new Biotronic generator. Rev. Bras. Cir. Cardiovasc., 9 (1): 40-53, 1994.

ABSTRACT: In the cardiomyoplasty, the contraction of the skeleton muscle, undergone electric stimulation on the dilated ventricle wall, increases the ventricle function, which is dependent of the heart and the base disease previous conditions. One of the main problems which interferes with the sucess of the cardiac muscle replacement is the myocardial synchronic stimulation and the skeleton muscle. The stimulation of this muscle in long-term has been possible because of the special electrodes associated with the sequential progressive stimulation, adjusting it to the cardiac function, through the gradual transformation of glicolitic fibers exposed to fatigue in highly resistant slow oxidizers. The pulse generator Myos (Biotronik) has been used in our center for electrical stimulation of the latissimus dorsalis in synchronization with the myocardium. This electronic circuit and lithium battery type of cardiomyostimulator stores a stimulation program responsible for different operational modes, adapted by a computer program. In order to program the cardiomyostimulator, the moment of the pulse synchronization with the aortic valve opening is extremely important. The high velocity $\mathrm{M}$ mode is utilized to evaluate the synchronism. The cardiomyoplasty clinical evaluation is based on the results obtained from 32 patients at $22 \mathrm{ti} 72$ years old (average $=46.2$ years) most of patients $(72 \%)$ presented dilated myocardiopathy due to undetermined cause, $24 \%$ of chagasic origin, $3 \%$ virus and $3 \%$ due to peripartum. Hospital and late mortality rate were both $12.5 \%$ and $3.1 \%$ and $3.7 \%$ respectively, excluding the chagasic patients. The actuarial survival was $81.3+-0.22 \%$ after 6 years and $94.4+-0.1 \%$ after 5 years, withdrawing the chagasic patients. The average indexes of systolic diameters $(55,1 \mathrm{~mm})$, diastolic $(70,7 \mathrm{~mm})$, segmental shortening $(22.8 \%)$ and ejection fraction $(51.0 \%)$, related to an average follow-up of 14.2 months, reflect that the cardiomyoplasty can be effective to assist the left ventricle. Choosing the right patient seems to be key to the goog operative result in short and long terms.

DESCRIPTORS: cardiomyoplasty; cardiomyostimulator; latissimus dorsalis.

\section{REFERÊNCIAS BIBLIOGRÁFICAS}

1 ACKER, M. A.; ANDERSON, W. A.; HAMMOND, R. L. - One to eleven weeks experience. $J$ Thorac. Cardiovasc. Surg., 94: 613-674, 1987.

2 AL-AMOLEND, W.; BULLER, A.; POPE, R. - Long term stimulation of cat fast-twitch skeletal muscle. Nature, 244: 227, 1973.

3 ARMENTI, F.; BITTO, T.; MACOVIAL, J. - Transformation of canine diaphragm to fatigue resultant muscle by phrenic nerve stimulation. Surg. Forum, 35: 258, 1984.

4 BRAILE, D. M.; SOARES, M. J. F.; RODRIGUES, M. C. Z.; RAMIN, S. L.; THEVENARD, R. S.; ZAIANTCHICK, M.; SOUZA, D. R. S. - Cardiomioplastia: estudo clínico de 26 pacientes em 6 anos. Rev. Bras. Marcapasso Arritmia, 6: 71-83, 1993.

5 BULLER, A.; ECCLES, J.; ECCLES, R. - Differentiation of fast and slow muscles in the cat hind limb. J. Physiol, 150: 399, 1960.

6 BULLER, A.; ECCLES, J.; ECCLES, R. - Interaction between motor nervous and muscles in respect of the characteristic speeds of their responses. $J$. Physiol, 150: 417, 1960.
7 CARPENTIER, A. \& CHACHQUES, J. C. - Latissimus dorsi cardiomyoplasty to increase cardiac output. In: RABAGO, G. \& COOLEY, D. A. (eds). Heart valve replacement: current status and future trends. New York, Futura Publishing, 1987. p. 473-486.

8 CARPENTIER, A. \& CHACHQUES, J. C. - Myocardial substitution with a stimulated skeletal muscle: first successful clinical case (letter). Lancet, 1: 1267,1985.

9 CARPENTIER, A. C. \& CHACHQUES, J. C. - Successful cardiomyoplasty with an electrostimulated latissimus dorsi muscle flap. In: Proc, 14th Meeting of the Neurolletric Society. Greece, Neurolletric Society, 1987, p.27.

10 CARPENTIER, A.; CHACHQUES, J. C.; ACAR, C. Dynamic cardiomyoplasty at seven years. J. Thorac. Cardiovasc. Surg., 106: 42-54, 1993.

11 CHACHQUES, J. C. - Effect of latissimus dorsi dynamic cardiomyoplasty on ventricular function. Circulation, 78: 203-216, 1988

12 CHACHQUES, J. C. \& CARPENTIER, A. - Postoperative management. In: CARPENTIER, A., CHACHQUES J-C \& GRANDJEA P. (eds.) Cardiomyoplasty. New York, Futura Publishing, 1991, p. 131-146. 
BRAILE, D. M.; SOARES, M. J. F.; SOUZA, D. R. S.; SCHALDACH, M. - Cardiomioplastia: novo gerador da Biotronic. Rev. Bras. Cir. Cardiovasc., 9(1): 40-53, 1994.

CHACHQUES J. C.; GRANDJEAN, P. A.; CARPENTIER, A. P. - Patient managment and clinical follow-up after cardiomyoplasty. J. Cardiac. Surg., 6: 89-99, 1991.

CHACHQUES, J.; GRANDJEAN, P.; VASSEUR, B. Electrophysiological conditioning of latissimus dorsi muscle flap for myocardial assistance. Artif Organs, 9: 27, 1985.

CHEKANOV, V. \& KRAKOVSKY, A - Possibilities of transformation of skeletal muscle. In: CHEKANOV V; KRAKOVSKY A. (eds). Cardiomyoplasty. Moscou, 1989. p. 22-38.

DEWAR, M. L. \& CHIU, R. C. - Cardiomyoplasty and the pulse-train stimulator. In: CHIU, R. C. - Biomechanical cardiac assist cardiomyoplasty and muscle-powered devices. New York, Futura Publishing, 1986. p. 4357.

17 DEWAR, M. L.; DRINKWATER, D. C.; CHIU, R. C. Synchronously stimulated skeletal muscle graft for myocardial repair. J. Thorac. Cardiovasc. Surg., 87: 325,1984

DIAZ, J. H. - Miocardioplastia: experiência cubana. 1990. Tese Doutorado. Havana, Instituto de Cardiologia.

DRINKWATER, O.; CHIU, R. C.; MODY, D. - Cardiac assist and myocardial repair with synchronously stimulated skeletal muscle. Surg. Forum., 31:271, 1980.

DUMCIUS, A.; SALCIUS, K.; GIEDRAITIS, S.; SIRVINSKAS, E.; KIBISA, R.; SKUCAS, J. Myoventriculoplasty with the use of programmed physiologically controlled electroneuro-stimulation (letter). J. Thorac. Cardiovasc. Surg., 97: 636-637, 1989.

21 GAINES, W. E.; GOLDBERG, N. H.; MERGNER, W. J. - Reconstruction of the right ventricular outflow tract with a revascularized free flap of striated muscle. Surg. Forum, 36: 250-253, 1985.

GRANDJEAN, P. A. - Electrical stimulation of skeleta muscles. In: CARPENTIER, A.; CHACHQUES, J-C.; GRANDJEAN, P. (eds.) Cardiomyoplasty. New York, Futura Publishing, 1991. p. 39-62.

HUME, W. I. - Construction of a functioning accessory miocardium. Trans. South. Surg. Assoc., 79: 200202, 1968.

JATENE, A. D.; MOREIRA, L. F. P.; STOLF, N. A. G. - Left ventricular function changes after cardiomyoplasty in patients with dilated cardiomiopathy. $J$. Thorac. Cardiovasc. Surg., 102: 132-139, 1991.

KANTROWITZ, A. \& McKINNON, W. - The experimental use of the diaphragm as an auxiliary myocardium. Surg. Forum, 9: 266-268, 1959.

diaphragmatic graft for augmenting LV function: a flexibility study. Trans. Am. Soc. Artif. Inter. Organs, 19: $251,1973$.

LEINER, A. A.; MOREIRA, F. P.; CHAGAS, A. C. P. Biomechanical circulatory assistance: importance of aerobic capacity of normal and conditioned skeletal muscles. Trans. Am. Soc. Artif. Intern. Organs, 34: 716-720, 1988.

33

MACOVIAK, J.; STEPHENSON, L. W.; ALAVI, A. A. Effect of electrical stimulation on diaphragmatic muscle used to enlarge right ventricle. Surg., 90: 271,1981

MACOVIAK, J.; STEPHENSON, L. W.; SPIELMAN, S. - Electrophysiological and mechanical characteristics of diaphragmatic autograft used to enlarge right ventricle. Surg. Forum, 31: 270, 1980.

MANION, J. F.; ACKER, M. A.; MAMMOND, R. L.; FALKMEYER, W.; DUCKET, S.; STEPHENSON, N. - Power output of skeletal muscle ventricles in circulation: short-term studies. Circulation, 76: 155$162,1987$.

MANNION, J. F. \& STEPHENSON, L. - Potencial uses of skeletal muscle for myocardial assistance. Surg. Clin. N. Am., 65: 679-687, 1985.

MOLTENI, L.; ALMADA, H.; FERREIRA, R. - Synchronously stimulated skeletal muscle graft for left ventricular assistance. J. Thorac. Cardiovasc. Surg., 97: 439-446, 1989.

$$
\begin{aligned}
& \text { - Resultados da cardiomioplastia no tratamento da } \\
& \text { vasc., 8: 85-95, } 1991 .
\end{aligned}
$$

MOREIRA, L. F. P.; BOCCHI, E. A.; SEFERIAN, P. J. - Influência da cardiomioplastia na sobrevida de pacientes portadores de cardiomiopatia dilatada. Arq. Bras. Cardiol., 57: 147-150, 1991.

MOREIRA, L. F.; SEFERIAN Jr. P.; BOCCHI, E. A. Survival improvement with dynamic cardiomyoplasty in patients with dilated cardiomyopathy. Circulation, 84 (Supl. 3): III-296-III302, 1991.

MOREIRA, L. F. P.; STOLF, N. A. G.; JATENE, A. D. - Benefits of cardiomyoplasty for dilated cardiomyopathy. J. Thorac. Cardiovasc. Surg., 3: 140-144, 1991.

PAPP, M. D. - Experimental use of intercostal muscle flaps for repair of induced cardiac defects. J. Thorac. Cardiovasc. Surg., 90: 261-264, 1985.

PETTE, O. \& HEILMAN, C. - Transformation of morphological, functional and metabolic properties of fast-twitch muscle as induced by long-term electrical stimulation. Basic Res. Cardiol., 72: 247-252, 1977. 
programmed stimulation and denervation on the histochemistry of skeletal muscle fiber types. Exp. Neurol., 40: 391, 1973.

40 ROSSI, M. A.; BRAILE, D. M.; SOUZA, D. R. S. Dynamic cardiomyoplasty in chronic Chagas heart disease: clinicopathological data. Ann. Thorac. Surg., 51: 649-651, 1991.

41 RUGGIERO, R.; POCHETTINO, A.; HAMMOND, R. L. - Autologous skeletal muscle, an alternative for cardiac assistance. Pace, 14: 480-491, 1991.

SALMONS, S. - An implantable muscle stimulator. $J$. Physiol., 188: 13, 1967.

43 SALMONS. S. - The response of skeletal muscle to different patterns of use. In: PETTE, D. (ed.) Plasticity of Muscle. New York, Walter de Gruyter, 1980. p. 387.

SALMONS, S. \& HENRIKSSON, J. - The adaptative response of skeletal muscle to increased use. Muscle Nerve, 4: 94, 1981.
SALMONS, S. \& VRBOVA, G. - The influence of activity on some contractile characteristics of mammalian fast and slow muscles. J. Physiol., 201: 535, 1969.

46 SCHALDACH, M. G. \& BRAILE, D. M. - The ANS signal utilized to optimize surgical treatment of cardiomyopathies (no preto).

47 SRETER, F.; GERGELY, J.; SALMONS, S.; ROMANUL, F. C. A. - Synthesis by fast muscle of myosin characteristic of slow muscle in response to longterm stimulation. Nature, 241: 17, 1973.

48 STRETER, F.; PINTER, K.; JOLEZ, F. - Fast to slow transformation of fast muscles in response to long-term phasic stimulation. Exp. Neurol., 75: $95,1982$.

49 VON RECUM, A.; STEELE, J.; HAMADA, D.; KANTROWITZ, A. - Long-term stimulation of a diaphragm muscle pouch. J. Surg. Res., 23: 422, 1977. 